Rev. salud pública. 12 (1): 14-26, 2010

\title{
Exposición a órganoclorados por ingesta de leche pasteurizada comercializada en Cartagena, Colombia
}

\section{Estimating exposure to organochlorine compounds from consuming pasteurised milk in Cartagena, Colombia}

\author{
Yolanda Castilla-Pinedo ${ }^{1}$, Luís Alvis-Estrada ${ }^{2}$ y Nelson Alvis-Guzmán ${ }^{2}$ \\ 1 Programa de Ingeniería de Alimentos. Universidad San Buenaventura Sede Cartagena. Colombia. \\ ycastilla@usbctg.edu.co \\ 2 Departamento de Investigaciones Económicas y Sociales, Facultad de Ciencias Económicas. \\ Universidad de Cartagena. Colombia.nalvis@yahoo.com
}

Recibido 2 Junio 2009/Enviado para Modificación 16 Enero 2010/Aceptado 2 Febrero 2010

\section{RESUMEN}

Objetivo Estimar la exposición de pobladores adultos a compuestos órganoclorados a partir de la ingesta de leche pasteurizada comercializada en la ciudad de Cartagena. Métodos Estudio observacional descriptivo de 47 muestras de lotes de producción de distintas marcas de leche pasteurizada y comercializada en la ciudad de Cartagena. El contenido de materia grasa en las muestras de leche fue determinado por Cromatografía de gases. Asimismo, fueron encuestadas 596 personas mayores de edad de las cuales se indagó la frecuencia de consumo de productos lácteos con el fin de estimar la ingesta diaria potencial y admisible y evaluar el riesgo a ingesta de organoclorados.

Resultados Las personas encuestadas se encuentran expuestas a compuestos órganoclorados mediante la ingesta de $12,1 \mathrm{mg} / \mathrm{g} / \mathrm{día}$ a través del consumo de leche pasteurizada comercializada en la ciudad de Cartagena. El $100 \%$ de las muestras analizadas se encontró contaminada con alguno de los plaguicidas objeto de estudio. La población objeto de estudio presenta una ingesta diaria (IDP) de compuestos órganoclorados superior a lo permitido, a la luz de la FAO/OMS.

Conclusiones No es posible recomendar a la población cartagenera no consumir leche, pero si recomendar a las autoridades competentes encargadas de exigir a las productoras que inicien campañas de mejoramiento de la calidad de la leche desde el punto de vista toxicológico.

Palabras Clave: Insecticidas organoclorados, exposición a compuestos químicos, leche, cromatografía de gases (fuente: DeCS, BIREME).

\section{ABSTRACT}

Objective Estimating adult residents' exposure to organochlorine compounds from drinking milk being marketed in the city of Cartagena.

Methods This was a descriptive observational study; 47 samples were taken from different production lots of brands of pasteurised milk being sold in the city of Cartagena. 
The milk samples' fat content was determined by gas chromatography. 596 adults were also surveyed whose dairy product consumption frequency had been investigated to estimate potential and acceptable daily intake and assess the risk of consuming organochlorines.

Results The people surveyed here were exposed to organochlorine compounds through consuming $12.1 \mathrm{mg} / \mathrm{g} /$ day in pasteurised milk being sold in the city of Cartagena. $100 \%$ of the samples tested were contaminated with one of the pesticides being studied. The study population had a daily intake (IDP) of organochlorine compounds greater than that permitted by FAO/WHO guidelines.

Conclusion It cannot be recommended that people in Cartagena do not drink milk; however, the competent authorities can be requested to require dairy-product producers to launch campaigns to improve the quality of milk from a toxicological point of view.

Key Words: Insecticide, organochlorine, chemical compound exposure, milk, gas chromatography (source: MeSH, NLM).

$\mathrm{L}$ a presencia de plaguicidas órganoclorados en la leche del ganado bovino se debe a sus propiedades fisicoquímicas de persistencia, liposolubilidad y bioacumulación así como al uso excesivo de estos compuestos en las prácticas agropecuarias $(1,2)$.

Los alimentos de origen animal como leche y carne se consideran la mayor fuente de residuos de estos plaguicidas en la alimentación humana. Diversos estudios registran presencia de los mismos plaguicidas en la leche humana (3-15) y han permitido confirmar la toxicidad de estos compuestos, afirmando que inducen actividad enzimática mediante radicales libres, alteran la respuesta inmunológica, afectan los procesos reproductivos, alteran el metabolismo lipídico, el transporte de vitaminas y de glucosa, además de que algunos son considerados mutagénicos, teratogénicos o carcinogénicos no sólo en la especie humana sino en las comunidades bióticas con diferente nivel de sensibilidad (16-19).

Aunque en Colombia se ha prohibido el uso de muchos compuestos órganoclorados como el DDT, Aldrín, Dieldrín, Endrín y restringido el uso de Lindano y de $\mathrm{HCH}$, aún se siguen encontrando residuos de estos compuestos en alimentos, tal y como lo muestra la presente investigación. Dada la importancia tóxica de estos compuestos, y de su continuo uso en hatos donde pasta el ganado productor de leche que se comercializa en la ciudad de Cartagena, se consideró pertinente conocer en 2 marcas comerciales de leche pasteurizada, el contenido de Heptacloro, $\mu \mathrm{g} / \mathrm{L}$, Lindano, Aldrín, Dieldrín, Heptacloro, Epóxido de heptacloro, Endrín, p, p'-DDT y sus metabolitos, para relacionarlos con los Límites Máximos de Residuos (LMR) que ha propuesto la FAO/OMS (20). 
No es solo la presencia de órganoclorados sino la cantidad en que se encuentran lo que constituye un problema de salud pública, pues a nivel mundial la ausencia total de plaguicidas no existe, sino por el contrario se han establecido LMR para los mismos, es decir, la concentración máxima de residuos de plaguicidas expresada en $\mathrm{mg} / \mathrm{Kg}$., tal como dice la comisión del Codex Alimentarius, para que se permita legalmente su uso en la superficie o parte interna de productos alimenticios de consumo humano.

El problema aumenta cuando la ingesta diaria posible (IDP) es superior a la ingesta diaria admisible (IDA), es decir, cuando el consumo de estos compuestos tóxicos por el ser humano es superior a las concentraciones que se pueden consumir diariamente sin que llegaran a ocasionar daño alguno.

\section{MATERIALES Y MÉTODOS}

Estudio observacional descriptivo que valora la exposición a compuestos órganoclorados y su riesgo para la salud de la población objeto de estudio. La población objeto: mayores de 18 años de edad de hogares de barrios de estrato 1 , 2 y 3 de Cartagena. Con una probabilidad de ingerir leche de $50 \%$, un error absoluto del $4 \%$ (error relativo del $8 \%$ ) y un nivel de confianza del $95 \%$ se definió una muestra de 596 personas. Se utilizó un muestreo aleatorio por conglomerado donde el conglomerado son los barrios. De dicha muestra se estimó la frecuencia de consumo de productos lácteos de las marcas comerciales de estudio a través de una Encuesta de frecuencia de consumo diseñada con la metodología de Frecuencia de Consumo y Diario Nutricional (21).

Los productos lácteos incluidos fueron de leche pasteurizada y perteneciente a las marcas comerciales. Posteriormente se llevó los gramos de yogur y queso a gramos de leche y se sumaron a los gramos de leche líquida consumidas. Solo se incluyeron en el estudio personas que consumen leche con frecuencia y que dicha leche o producto lácteo pertenezca a una de las dos marcas comerciales de leche que son objeto de estudio.

Caracterización de la leche

Se tomaron 47 muestras (unidades en bolsas de $1 / 2$ litro que en su etiqueta llevaban el número del lote de producción) de leche pasteurizada de dos marcas locales. Las muestras se recolectaron en 30 días, a partir de la primera semana del mes de Septiembre. A las muestras se recolectaron en condiciones asépticas y congeladas inmediatamente y liofilizadas antes de 48 horas. El contenido de materia grasa, fue determinado por Cromatografía de gases, (equipo Cromatografía de gases Agilemt, modelo 48900, con detector de microcaptura de electrones). 
Los compuestos órganoclorados (DDT, Aldrín, Dieldrin, Lindano, Hexaclorociclohexano $(\mathrm{a}+\mathrm{b})$, Heptacloro, Endrin Hepoxido de heptanocloro y Endrin), se cuantificaron de acuerdo al método recomendado por la Agencia de Protección Ambiental de los Estados Unidos (EPA) es decir la Cromatografía de gases, para determinación detallada de esta técnica.

\section{Procedimientos}

Extracción. A $100 \mathrm{ml}$ de leche pasteurizada se le ajustó el pH entre 5 y 7 y se adicionaron $5 \mathrm{ml}$ de metano; Fueron pasados a través de cartucho de extracción en fase sólida (SPE) C-18, el cual previamente fue acondicionado con $3 \mathrm{ml}$ de metanol seguida de $2 \mathrm{ml}$ de metanol en agua al $2 \%$. La extracción de los pesticidas fue realizada con 2 volúmenes de $4 \mathrm{ml}$ de una mezcla de hexano:cetona (90:10). El eluato final fue luego concentrado a presión reducida hasta un volumen final de $1 \mathrm{ml}$. Las muestras fueron extraídas por triplicado.

Determinación cromatográfica. Se incorporó una columna capilar SPB-5 de $30 \mathrm{~m} \times 0.25 \mathrm{~mm}$. D.I. x $0.25 \mathrm{~mm}$ de espesor de capa. Fase estacionaria fenilmetilsilicon al $5 \%$, volumen de muestra $1-^{\mu}$ tiempo de Split 0.75 min. Condiciones de operación: temperatura inyector $260^{\circ} \mathrm{C}$; temperatura detector $320^{\circ} \mathrm{C}$; Gas de arrastre helio a $1 \mathrm{~mL} / \mathrm{min}$; Gas auxiliar Nitrógeno a $30 \mathrm{~mL} / \mathrm{min}$. Programa de temperatura: inicial de $90^{\circ} \mathrm{C}$ durante $2 \mathrm{~min}$.; Incremento de $30^{\circ} \mathrm{C} /$ min., hasta $180^{\circ} \mathrm{C}$; Incremento de $1^{\circ} \mathrm{C} / \mathrm{min}$, hasta $200^{\circ} \mathrm{C}$; Luego incremento de $10^{\circ} \mathrm{C} / \mathrm{min}$. Hasta $300^{\circ} \mathrm{C}$ y mantenida a esta temperatura final por $3 \mathrm{~min}$.

Análisis cuantitativo. Se realizó empleando el método de estándar externo, para lo cual se utilizó una mezcla estándar de órganoclorados de Chem Service (CSM-8880 M. West Chester, PA 19381-0599.USA), de una concentración de $20 \mathrm{ng} / \mathrm{ml}$ de cada componente, y los resultados se expresaron en $\mu \mathrm{g} / \mathrm{l}$ de grasa anhidra. La confirmación de los compuestos POs analizados se realizó, cuando fue necesario, en un equipo GC-MS marca Finigan 5475 Mat CGQ con sistema de ionización electrónica que opera a 70 eV. Las pruebas de recuperación fueron iguales o superiores al $80 \%$, según lo recomendado por FIL-IDF (22). La linealidad en la calibración presentó coeficientes de correlación entre 0,9925 y 0,9992; en las pruebas de repetibilidad se obtuvo valores de desviación estándar entre 0,35 1,78. Los coeficientes de variación fluctuaron entre 1,65 y 14,82; los límites de detección se presentaron en el rango 0,0001 y el límite de cuantificación de 0,02 microgramo por litro (23). 
Determinación de la IDP y posterior evaluación de riesgo

Para determinar la ingesta diaria admisible-IDA, fue preciso hallar la ingesta diaria posible (IDP) de compuestos órganoclorados de acuerdo a la legislación colombiana vigente o a lo establecido por el Codex Alimentarius, FAO y OMS (20). Luego, a partir del diario nutricional de leche y mediante la cuantificación del contenido de compuestos órganoclorados, se estimó la ingesta diaria posibleIDP, la cual es igual a un Gramo de Leche Consumido/Día X Mg de Organoclorado/Gramo de leche).

Se indagó el peso de los encuestados y se corroboró pesándolos en una balanza para uso domestico (Sankey Bathroom Scale Modelo kg 115). Se indagó la frecuencia de consumo mensual de los productos lácteos de las marcas en estudio. Asimismo, se obtuvo el promedio de gramos que ingieren cada vez que consumen. Luego se multiplicó el número de veces que consumen al mes los productos lácteos por la cantidad en gramos y posteriormente se dividió entre 30, hallando los gramos diarios consumidos.

La evaluación de la exposición a compuestos órganoclorados está basada en recomendaciones de varias instituciones científicas, las cuales establecen los rangos de ingestión de compuestos órganoclorados (IDA) (24):

1. IDP < $30 \%$ de la IDA: Riesgo bajo.

2. IDP entre 30 a $100 \%$ de la IDA: Riesgo medio.

3. IDP > $100 \%$ de la IDA: Riesgo alto.

Ingesta total de órganoclorados se determinó mediante: $\operatorname{It}(\mathrm{OCS})_{\mathrm{i}}=\mathrm{C}_{\mathrm{i}} \mathrm{V}_{\mathrm{i}}$, es decir a la concentración de organoclorados en el elemento ingerido, $\mathrm{mg} / \mathrm{ló} \mathrm{mg} / \mathrm{kg}$ multiplicado por la cantidad del elemento ingerido, $1 / \mathrm{d}$ o kg/d.

La dosis de exposición a órganoclorados está dada por $\mathrm{DEj}$, la cual determinó usando la siguiente ecuación (siguiendo los criterios de Ortiz) (25):

$$
D E_{j} \approx \frac{\sum_{i} C_{i} V_{i}}{W_{j}}
$$

Es decir, la sumatoria de la ingesta total de órganoclorados dividida entre el peso de la persona. 


\section{RESULTADOS}

El queso es el lácteo más consumido por los encuestados (solo el $3 \%$ de la población no consume queso), seguido de la leche con un $95 \%$ y por último el yougourt el cual es consumido por uno de cada tres encuestados (Tabla 1).

Tabla 1. Consumo de queso, leche y yogurt entre los encuestados

\begin{tabular}{lcccc}
\hline & Consumo & $\mathrm{N}$ & $\%$ & $\begin{array}{c}\text { Promedio de gramos } \\
\text { consumidos al día }\end{array}$ \\
\hline Queso & $\mathrm{No}$ & 17 & 2,9 & \\
Leche & $\mathrm{Si}$ & 579 & 97,1 & 74,5 \\
& $\mathrm{No}$ & 32 & 5,4 & \\
\multirow{5}{*}{ Yogurt } & $\mathrm{Si}$ & 564 & 94,6 & 223,5 \\
& $\mathrm{No}$ & 150 & 25,2 & 38,8 \\
& $\mathrm{Si}$ & 446 & 74,8 & \\
\hline
\end{tabular}

En las 47 muestras analizadas se puede observar que la razón(X/LMR) de los plaguicidas $\gamma$ - BHC, $\alpha$-Clordano, Endrin y Endrin Cetona están por encima de 1 . El valor promedio de órganoclorados encontrado por cada muestra fue de 0,004122 $\mathrm{mg} / \mathrm{g}$. El Endosulfan II y Endosulfan Sulfato fueron los compuestos que presentaron menor concentración mientras que $\gamma$ - BHC y $\alpha$-Clordano reportaron mayores niveles de concentración. No se encontró DDT. Los compuestos $\gamma$ - Clordano, $\alpha$ Clordano y Metoxicloro reportaron los mayores porcentajes de muestras positivas (80,9 \%, 80,9 \% y 78,7 \% respectivamente) mientras que el Endosulfan Sulfato y Endosulfan II mostraron las menores frecuencias de muestras positivas $(2,1 \%$ y $4,3 \%$ respectivamente).

En cuanto a las Ingesta Diarias Admisibles-IDA ajustadas por peso, los compuestos con menores niveles son el Aldrin, Dieldrin, Endrin, Endrin aldehído y Endrin Cetona. Mientras que el Endosulfan I, Endosulfan II, Endosulfan Sulfato, DDT, DDE, y D.D.D presentan mayores niveles. Los compuestos que reportaron menor frecuencia de muestras con concentraciones por encima de las IDA ajustadas por peso fueron el Endosulfan II, Endosulfan Sulfato (con excepción del DDT que no se encontró en ninguna de las muestras). En contraste los compuestos $\gamma$ Clordano, $\alpha$-Clordano y $\delta$-BHC reportan las más altas frecuencias de muestras con concentraciones mayores que las IDA ajustadas por peso (Tabla 2).

Del mismo modo, elEndrin, Endrin aldehído, Endrin Cetona, $\alpha-\mathrm{BHC}, \beta-\mathrm{BHC}$, $\gamma$-BHC, $\delta$-BHC reportan menores límites máximos residuales- LMR en contraste con el Endosulfan I, Endosulfan II, Endosulfan Sulfato, DDE, D.D.D los cuales presentan limites de mayor valor. Es así como el Endrin Cetona seguido del Endrin 
reportan la mayor proporción de muestras cuyas concentraciones se encuentran por encima de los Límites Máximos Residuales -LMR mientras que en las muestras no se encontraron niveles residuales no permitidos de compuestos de $\alpha$ - BHC, $\beta$ BHC, DDT, DDE, D.D.D, Endosulfan I, Endosulfan II, Endosulfan Sulfato (Tabla 2).

Los heptacloros $(\alpha+\gamma+\delta)$-BHC se presentan en mayor porcentaje en la marca $\mathrm{B}$, exceptuando el $\beta$-BHC que es de mayor concentración en la marca A. Es de resaltar la ausencia del DDT en cada una de las marcas estudiadas, no siendo así con sus metabolitos; El DDE se presenta en mayor porcentaje en la marca A, correspondiente a 30,4\% de las muestras analizadas, mientras que para el DDD es mayor en la marca $\mathrm{B}$ con un porcentaje del $16,7 \%$ en relación con el de la marca $\mathrm{B}$ que es de un $13 \%$. Los compuestos órganoclorados que en mayor porcentaje presentaron resultado positivo fueron Heptacloro epóxido, $\gamma$ - Clordano, $\alpha$ - Clordano, con un porcentaje de 87,5\% de muestras en las cuales estuvieron presentes todos en la marca B. El aldrín dio un porcentaje de muestras positivas superior en la marca B, correspondiente al 66,7 \% mientras que para la marca A este mismo compuesto fue de $60,9 \%$. Para caso del dieldrín su presencia fue mayor en la marca A con un porcentaje de muestras positivas de 26,3\% (Tabla 2).

En la leche de la marca A, se observa que el contenido de lindano, aldrín + dieldrín y DDT + metabolitos están por debajo de la norma FAO/OMS (3), ya que los valores de X/LMR son menores que 1 . Sin embargo, $\gamma$ - BCH y endrín alcanzan valores X/LMR aproximados de 26,3 y 1,3, respectivamente (Tabla 3).

Evaluación del riesgo. Relación entre la IDP y la IDA ajustada por peso. El promedio IDP de órganoclorados en leche encontrado en la población de estudio fue de 1,298 g/g/día. Las menores concentraciones de IDP se dieron a través del DDT, Endosulfan II y Endosulfan Sulfato. Mientras que el compuesto con mayor IDP fue $\gamma$ - BHC seguido de $\alpha$-Clordano (Tabla 4).

Se identifico como riesgo alto de consumo, los compuestos $\gamma$-BHC, $\gamma$-Clordano, $\alpha$-Clordano, Endrin aldehido y Endrin Cetona. Mientras que de riesgo medio $\delta$ BHC, Aldrin, Diledrin y Endrin.. El resto de los compuestos presentaron un riesgo bajo. Las razones IDP/IDA más altas se encontraron en los compuestos $\gamma$ - BHC y $\gamma$-Clordano (Tabla 4).

La dosis de exposición en la población de estudio se estimo cerca de 12,080 g/ g/día. Siendo las dosis de exposición más altas en los compuestos $\gamma$-BHC y $\gamma$ Clordano (7,707 g/g/día y 2,638 g/g/día) (Tabla 4). 
Tabla 2. Residuos de plaguicidas órganoclorados presentes en muestras de leche pasteurizada de dos marcas comerciales. Cartagena de Indias (mg/g base grasa, $\mathrm{n}=47$ )

\begin{tabular}{|c|c|c|c|c|c|c|c|}
\hline Plaguicida & $X(\mathrm{mg} / \mathrm{g})$ & $\begin{array}{c}\% \text { de } \\
\text { muestras } \\
\text { positivas }\end{array}$ & $\begin{array}{l}\text { IDA } \\
(g / g)\end{array}$ & $\begin{array}{l}\text { LMR } \\
(\mathrm{mg} / \mathrm{g})\end{array}$ & $\begin{array}{c}\% \text { de } \\
\text { muestras } \\
\text { >LMR }\end{array}$ & $\begin{array}{c}\% \text { de } \\
\text { muestras } \\
>\text { IDA }\end{array}$ & X'LMR \\
\hline$\alpha-B H C$, & 0,00001 & 31,9 & 0,0000008 & 0.0001 & 0 & 31,9 & 0.100 \\
\hline$\beta-B H C$, & 0,00001 & 34 & 0,0000008 & 0,0001 & 0 & 34 & 0,100 \\
\hline $\mathrm{Y}-\mathrm{BHC}$ & 0,00263 & 53,2 & 0,0000008 & 0,0001 & 21,3 & 100 & 26,300 \\
\hline $\bar{\delta}-\mathrm{BHC}$ & 0,00005 & 72,3 & 0,0000008 & 0,0001 & 6,4 & 72,3 & 0,500 \\
\hline Heptacloro & 0,00002 & 70,2 & 0,0000005 & 0,00015 & 2,1 & 70,2 & 0,133 \\
\hline $\begin{array}{l}\text { Heptacloro } \\
\text { epóxido }\end{array}$ & 0,00001 & 76,6 & 0,0000005 & 0,00015 & 6,4 & 76.6 & 0.067 \\
\hline Aldrin & 0,00002 & 63,8 & 0,0000001 & 0,00015 & 0 & 63,8 & 0,133 \\
\hline Dieldrin & 0,00002 & 23,4 & 0,0000001 & 0,00015 & 4,3 & 23,4 & 0,133 \\
\hline Y-Clordano & 0,00012 & 80,9 & 0,0000005 & 0,00015 & 27,7 & 80,9 & 0,800 \\
\hline a-Clordano & 0,00090 & 80,9 & 0,0000005 & 0,00015 & 17 & 80,9 & 6,000 \\
\hline DDT-------. & 0.000 & 0 & 0,00002 & 0,00125 & 0 & 0 & 0.000 \\
\hline DDE, & 0,00001 & 23,4 & 0,00002 & 0,00125 & 0 & 19,1 & 0.008 \\
\hline D.D.D, & 0,00001 & 14,9 & 0,00002 & 0,00125 & 0 & 12,8 & 0.008 \\
\hline Endosulfan I & 0,00004 & 40,4 & 0,000006 & 0,0004 & 0 & 40,4 & 0,100 \\
\hline Endosulfan II & 0,000001 & 4,3 & 0,000006 & 0,0004 & 0 & 2,1 & 0,003 \\
\hline $\begin{array}{l}\text { Endosulfan } \\
\text { Sulfato }\end{array}$ & 0,000002 & 2,1 & 0,000006 & 0,0004 & 0 & 2,1 & 0,005 \\
\hline Endrin & 0,000027 & 29,8 & 0,0000002 & 0,00002 & 29,8 & 29,8 & 1,350 \\
\hline $\begin{array}{l}\text { Endrin } \\
\text { aldehído }\end{array}$ & 0,000009 & 14,9 & 0,0000002 & 0,00002 & 14,9 & 14,9 & 0,450 \\
\hline $\begin{array}{l}\text { Endrin } \\
\text { Cetona }\end{array}$ & 0,000042 & 59,6 & 0,0000002 & 0,00002 & 55,3 & 59,6 & 2,100 \\
\hline Metoxicloro & 0,000191 & 78,7 & N.D. & N.D. & N.D. & N.D. & N.D. \\
\hline $\mathrm{C}$ & 0,004122 & & & & & & \\
\hline
\end{tabular}

Tabla 3. Proporción de muestras positivas con residuos de plaguicidas órganoclorados, en muestras de leche pasteurizada discriminado en las dos marcas comerciales, Cartagena de Indias ( $\mathrm{mg} / \mathrm{g}$ base grasa, $\mathrm{n}=47$ )

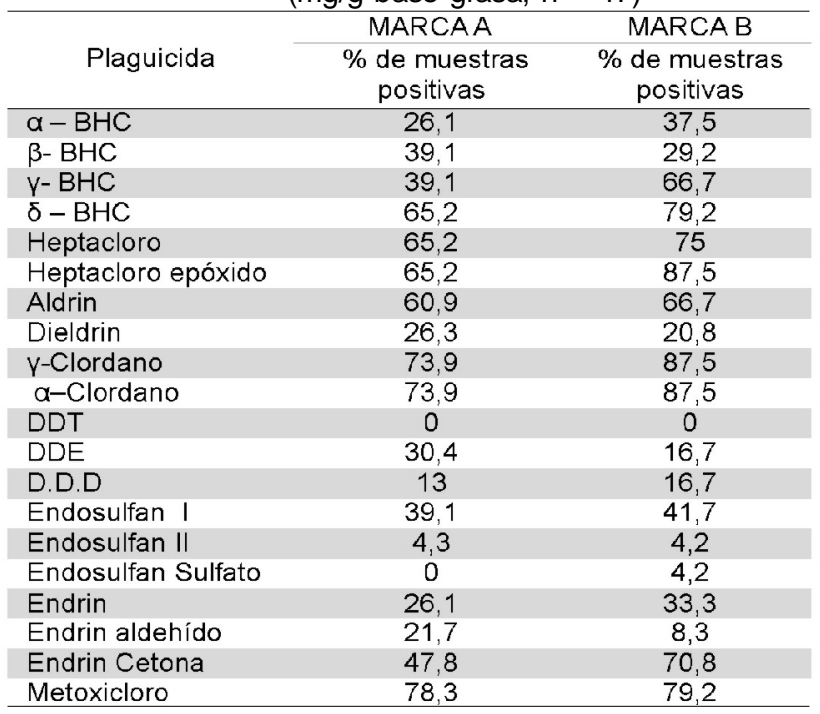


Tabla 4. Ingesta Diaria Posible-IDP. Promedio e Ingesta Diaria Admisible-IDA ajustada por peso- de residuos de plaguicidas órganoclorados presentes en muestras de leche pasteurizada de dos marcas comerciales. Evaluación del riesgo, razón entre IDP e IDA y Dosis de Exposición, Cartagena (mg/g base grasa, $n=47$ )

\begin{tabular}{|c|c|c|c|c|c|}
\hline & $\begin{array}{c}\text { IDP } \\
\text { promedio } \\
\text { (mg/gr/día) }\end{array}$ & $\begin{array}{l}\text { IDA promedio } \\
\text { ajustado por } \\
\text { peso } \mathrm{mg}\end{array}$ & $\begin{array}{l}\text { Evaluación del } \\
\text { riesgo }\end{array}$ & $\begin{array}{l}\text { Razón } \\
\text { IDP/IDA }\end{array}$ & $\mathrm{DE}$ \\
\hline IDP & 1,298 & & & & 12,080 \\
\hline $\mathrm{a}-\mathrm{BHC}$ & 0,003 & 0,05235571 & Riesgo Bajo & 0,06 & 0,029 \\
\hline$\beta-B H C$ & 0,003 & 0,05235571 & Riesgo Bajo & 0,06 & 0,029 \\
\hline $\mathrm{y}-\mathrm{BHC}$ & 0,828 & 0,05235571 & Riesgo Alto & 15,81 & 7,707 \\
\hline$\delta-\mathrm{BHC}$ & 0,016 & 0,05235571 & Riesgo Medio & 0,30 & 0,147 \\
\hline Heptacloro & 0,006 & 0,03272232 & Riesgo Bajo & 0,19 & 0,059 \\
\hline Heptacloro epóxi do & 0,003 & 0,03272232 & Riesgo Bajo & 0,10 & 0,029 \\
\hline Aldrin & 0,00600 & 0,00654446 & Riesgo Medio & 0,96 & 0,059 \\
\hline Dieldrin & 0,006 & 0,00654446 & Riesgo Medio & 0,96 & 0,059 \\
\hline y Clordano & 0,038 & 0,03272232 & Riesgo Alto & 1,15 & 0,352 \\
\hline a-Clordano & 0,283 & 0,03272232 & Riesgo Alto & 8,66 & 2,638 \\
\hline DDT & 0,000 & 1,30889267 & Riesgo Bajo & 0,00 & 0,000 \\
\hline $\mathrm{DDE}$ & 0,003 & 1,30889267 & Riesgo Bajo & 0,00 & 0,029 \\
\hline DDD & 0,003 & 1,30889267 & Riesgo Bajo & 0,00 & 0,029 \\
\hline Endosulfan I & 0,013 & 0,3926678 & Riesgo Bajo & 0,03 & 0,117 \\
\hline Endosulfan II & 0,000 & 0,3926678 & Riesgo Bajo & 0,00 & 0,000 \\
\hline Endosulfan Sulfato & 0,001 & 0,3926678 & Riesgo Bajo & 0,00 & 0,003 \\
\hline Endrin & 0,008 & 0,01308893 & Riesgo Medio & 0,65 & 0,006 \\
\hline Endrin aldehído & 0,028 & 0,01308893 & Riesgo Alto & 2,16 & 0,079 \\
\hline Endrin Cetona & 0,013 & 0,01308893 & Riesgo Alto & 1,01 & 0,264 \\
\hline Metoxicloro & 0,060 & N.D. & N.D. & N.D. & 0.123 \\
\hline
\end{tabular}

Se determinó que el $97 \%$ de la población estudiada presenta un riesgo alto de padecer algunos trastornos de salud por el consumo de leche contaminada con $\gamma$ - BHC; el $43 \%$ con el consumo de $\gamma$-Clordano; el $94 \%$-Clordano, el 68,7 \% con el consumo de leche con Endrin aldehido y el 39,7 \% tiene el mismo tipo de riesgo cuando consume Endrin Cetona, Considerándose un riesgo inaceptable, por lo que deberán reducirse inmediatamente las concentraciones encontradas.

Mientras que uno de cada tres encuestados presenta un riesgo Medio de padecer algún tipo de trastornos adversos de tipo crónico al consumir leche contaminada con $\delta$-BHC; El $44 \%$ tiene riesgo medio cuando consume Aldrin, Diledrin y Endrin. Bajo este nivel de riesgo el consumo de leche no traerá problemas a las personas adultas, pero deberá tomarse cierta precaución con los niños y con los consumidores exagerados. El resto de los compuestos analizados, constituyen para la población un riesgo bajo al consumir leche con estas sustancias, aunque bajo este nivel de riesgo no existe problemas para la salud, se debe tener cuidado sobre todo con los niños. 


\section{DISCUSIÓN}

En el presente estudio la estimación de la Ingesta Posible de Leche está fundamentada en un autoreporte brindado por las personas encuestadas. Al diligenciar la encuesta de consumo de productos lácteos (cuestionario basado en el Diario nutricional) las personas pueden no recordar la cantidad exacta consumida diariamente siendo posible que se presente un sesgo de memoria en algunas ellas. Se intentó reducir el riesgo de cometer este sesgo delimitando las preguntas a periodos de reciente recordación de manera parecida a estudios realizados en la misma temática $(26,27)$.

A pesar de que en Colombia se prohibió el uso de plaguicidas órganoclorados a mediados de los años 80, en especial el DDT, aldrin, heptacloro, dieldrin, clordano y canfecloro, y los compuestos a base de clordimeform (formamidina) o sus sales (28), aún se sigue encontrando residuos de estos plaguicidas en alimentos, como producto de su uso irracional. Este panorama es coherente con los resultados encontrados en la presente investigación. Debido a la característica lipofílica de algunos alimentos, tales compuestos se localizan de modo importante en la carne y en la leche. Por lo que se ha estimado el nivel de contaminación de la leche a partir del alimento de uso animal (18).

Los resultados cualitativos de órganoclorados determinados en la presente investigación, refleja el grado de contaminación de las muestras de leches analizadas ( $\mathrm{n}=47$ ), en la ciudad de Cartagena, concluyendo que todas estas contenían alguno de los plaguicidas objeto de estudio, lo cual contrasta con lo reportado por Vega y León (30) quienes encontraron que en 4 marcas de leche comercializadas en la ciudad de México el X/LMR fue superior a 1 .

La alta IDP de los compuestos $\gamma$ - BHC y $\alpha$-Clor en las muestras de las dos marcas de leche producidas y comercializadas en la ciudad de Cartagena se considera anómala con respecto a la resolución 10255 de 1993 del ministerio de salud de Colombia en la que se prohíbe la importación, producción, formulación, comercialización, venta, uso y manejo de productos con ingrediente activo de Clordano y BHC.

Las bajas concentraciones de IDP en los compuestos DDT, Endosulfan II y Endosulfan Sulfato resultan ser tranquilizantes y coherentes con la norma emitida por la resolución 891 de 1986 del ICA, la cual cancela licencia de ventas de productos de uso agrícola compuestos de DDT y mediante la emisión del decreto 305 de 1988 en la que se exceptúa el uso de Endosulfan solo para el tratamiento tratar broca del café. 
La resolución 10255 del Ministerio de Salud prohíbe en Colombia el uso de Clordano, $\gamma$-BHC mientras que la resolución 1849 prohíbe el Endrin Cetona y el Endrin. Estos compuesto fueron los que reportaron mayor proporción de muestras cuyas concentraciones superan los Limites Máximos Residuales -LMR recomendado por entidades internacionales como la FAO/OMS en contraste con lo reportado por Prado (3) los cuales registraron valores altos en la evaluación de $(\alpha+\beta)-H C H$, aldrín+dieldrín y Endrin, lo que presume el uso aún de estos compuestos en el control de plagas.

Compuestos como $\alpha$-BHC, $\beta$-BHC, DDT, DDE, D.D.D, Endosulfan I, Endosulfan II, Endosulfan Sulfato, presentaron valores de LMR por debajo de los niveles permitidos, resultados que contrastan con la normatividad vigente en Colombia en relación con la prohibición de la producción y uso de estos compuestos (dejando el Endosulfan solo para uso contra la Broca del Café).

La presencia de cantidades de Endosulfan Sulfato superiores a IDA en el $100 \%$ de las muestras de una de las marcas es semejante a lo encontrado por Prado (30). La presencia del heptacloro y el heptacloro epoxido $(54,5 \%$ y $58,3 \%$ de las muestras) está relacionada con el uso de este pesticida en insumos como maíz, granos, sorgo, cultivos que luego son empleados en la alimentación animal o en la elaboración de ensilajes, heno o concentrados alimenticios (31-33).

Se encontraron resultados semejantes a los de Izquierdo (34) en relación a la presencia de Dieldrin y Aldrin. Aunque en las muestras analizadas no se encontró DDT si se hallaron niveles de concentración de DDE y DDD mayores que la IDA. Esto es posible debido a lo lento que es la biodegradación del DDT.

La situación es alarmante si se tiene en cuenta que la población a la que se le hizo el estudio, son adultos mayores en donde el consumo promedio es alrededor de 314,8 g/día. Por lo que al calcular la ingesta diaria posible promedio IDP de órganoclorados fue de $1,3 \mathrm{mg} / \mathrm{g}$, superando la IDA promedio recomendada por la FAO/OMS (20), la cual fue de $0,00000442 \mathrm{mg} / \mathrm{g}$.

El riesgo de exposición a compuestos órganoclorados se asocia fundamentalmente con la exposición a largo plazo, a causa de su persistencia en el medio ambiente, acumulación de la grasa corporal y carcinogenicidad en animales en experimentación (35). Este estudio resulta similar en cuanto a los resultados encontrados por Duarte, en donde al estudiar pacientes con Cáncer y aplasia medular, se encontró en el tejido adiposo de estas personas algunos plaguicidas órganoclorados como el DDT total, Lindano, Clordano y BHC con valores de 
13,49- 0.071-0.634 y 0,029 en ppm respectivamente (36). Por lo anterior, es importante definir las rutas de exposición al DDT de la población en mayor riesgo, para plantear una estrategia que permita reducir el riesgo en salud \&

\section{REFERENCIAS}

1. Wong SK, Lee WO. Survey of organochlorine pesticide residues in milk in Hong Kong (19931995). J AOAC Int. $1997 \mathrm{Nov-Dec;80} \mathrm{(6):1332-5.}$

2. Pinto M, Montes L, Anrique R, Carrillo R, Tamayo R, Cristi R. Residuos de plaguicidas organoclorados en leche de vaca y su relación con alimentos para uso animal como fuentes de contaminación. Arch Med Vet. 1990;22:143-53.

3. Behrooz RD, Sari AE, Bahramifar N, Ghasempouri SM. Organochlorine pesticide and polychlorinated biphenyl residues in human milk from the Southern Coast of Caspian Sea, Iran. Chemosphere. 2009 Feb;74(7):931-7.

4. Burke ER, Holden AJ, Shaw IC, Suharyanto FX, Sihombing G. Organochlorine pesticide residues in human milk from primiparous women in Indonesia. Bull Environ Contam Toxicol. 2003 Jul;71(1):148-55.

5. Ennaceur S, Gandoura N, Driss MR. Organochlorine pesticide residues in human milk of mothers living in northern Tunisia. Bull Environ Contam Toxicol. 2007 May;78(5):325-9.

6. Kumar A, Baroth A, Soni I, Bhatnagar P, John PJ. Organochlorine pesticide residues in milk and blood of women from Anupgarh, Rajasthan, India. Environ Monit Assess. 2006 May;116(1-3):1-7.

7. Kumar A, Dayal P, Singh G, Prasad FM, Joseph PE. Persistent organochlorine pesticide residues in milk and butter in agra city, India: a case study. Bull Environ Contam Toxicol. 2005 Jul;75(1):175-9.

8. Nag SK, Raikwar MK. Organochlorine pesticide residues in bovine milk. Bull Environ Contam Toxicol. 2008 Jan;80(1):5-9.

9. Okonkwo JO, Kampira L. Organochlorine pesticide residues in mother's milk in Swaziland, 1996-1997. Bull Environ Contam Toxicol. 2002 May;68(5):740-6.

10. Pandit GG, Sahu SK. Assessment of risk to public health posed by persistent organochlorine pesticide residues in milk and milk products in Mumbai, India. J Environ Monit. 2002 Feb;4(1):182-5.

11. Pandit GG, Sharma S, Srivastava PK, Sahu SK. Persistent organochlorine pesticide residues in milk and dairy products in India. Food Addit Contam. 2002 Feb;19(2):153-7.

12. Pardio VT, Waliszewski KN, Landin LA, Bautista RG. Organochlorine pesticide residues in cow's milk from a tropical region of Mexico. Food Addit Contam. 2003 Mar;20(3):259-69.

13. Sanghi R, Pillai MK, Jayalekshmi TR, Nair A. Organochlorine and organophosphorus pesticide residues in breast milk from Bhopal, Madhya Pradesh, India. Hum Exp Toxicol. 2003 Feb;22(2):73-6.

14. Schinas V, Leotsinidis M, Alexopoulos A, Tsapanos V, Kondakis XG. Organochlorine pesticide residues in human breast milk from southwest Greece: associations with weekly food consumption patterns of mothers. Arch Environ Health. 2000 Nov-Dec;55(6):411-7.

15. Stuetz W, Prapamontol T, Erhardt JG, Classen HG. Organochlorine pesticide residues in human milk of a Hmong hill tribe living in Northern Thailand. Sci Total Environ. 2001 Jun 12;273(13):53-60.

16. Brent GA, Harney JW, Moore DD, Larsen PR. Multihormonal regulation of the human, rat, and bovine growth hormone promoters: differential effects of 3',5'-cyclic adenosine monophosphate, thyroid hormone, and glucocorticoids. Mol Endocrinol. 1988 Sep;2(9):792-8.

17. Durham PJ. Infectious bovine rhinotracheitis virus and its role in bovine abortion. N Z Vet $\mathrm{J}$. 1974 Oct;22(10):175-80. 
18. Heeschen W, Bluthgen A. Organochlorine compounds (including polychlorinated biphenyls (PCB)) and carry-over mechanisms. Dtsch Tierarztl Wochenschr. 1985 Jun 7;92(6):221-8.

19. Waliszewski SM, Aguirre AA, Infanzon RM, Siliceo J. Carry-over of persistent organochlorine pesticides through placenta to fetus. Salud Publica Mex. 2000 Sep-Oct;42(5):384-90.

20. FAO/OMS. Food and Agriculture Organization of the United Nations. Residuos de plaguicidas en los alimentos. Informe de la Reunión Conjunta 1981 del Cuadro de Expertos de la FAO en Residuos de Plaguicidas y el Medio Ambiente y el grupo de Expertos de la OMS en Residuos de Plaguicidas. Roma, Italia; 1982.

21. Hernandez-Avila M, Romieu I, Parra S, Hernandez-Avila J, Madrigal H, Willett W. Validity and reproducibility of a food frequency questionnaire to assess dietary intake of women living in Mexico City. Salud Publica Mex. 1998 Mar-Apr;40(2):133-40.

22. FILIDF. International Standard 75C. Milk and Milk products: Recomended Methods for Determination of Organochlorine Compounds (Pesticides). Brussels, Belgium; 1991.

23. Quattrocchi O, Abelaira De Andrizzi SI, Laba RF. Validación de métodos. In: Artes Gráficas Farro S.A. (Ed.). Introducción a la HPLC, Aplicación y Práctica. Buenos Aires, Argentina; 1992. p. 301-28.

24. Majul Eugenia M, Morón-Jiménez MJ, Ramón Adriana-Noemí. Estimación de la Ingesta Diaria Potencial de Nitritos en Productos Cárnicos de Mayor Consumo en Adolescentes. Revista de Salud Pública y Nutrición RESPYN. 2004 Julio- Septiembre; 5 (3). p. 17-26.

25. Ortiz D, Castro L, Turrubiartes F, Milan J, Diaz-Barriga F. Assessment of the exposure to fluoride from drinking water in Durango, Mexico, using a geographic information system. Fluoride. 1998 Nov;31(4):183-7.

26. Sebba MM, Merchán-Hamann E, da Cunha-Floresta AC. Prácticas alimentarias y razones para cambios en la alimentación de la población adulta de Brasilia. Rev Cubana Salud Pública 2005 octubre - diciembre; 31 (4) 15-23.

27. Tristan-Fernandez JM, Ruiz SF, Perez de la Cruz A, Lobo TG, Aguilar-Cordero MJ, ColladoTorreblanca $F$. The influence of nutrition and social environment on the bone maturation of children. Nutr Hosp. 2007 Jul-Aug;22(4):417-24.

28. Toro G. Hombre, Hambre y Contaminación del Medio Ambiente. Rev Fac Med Univ Nal Colomb. 1993;41(1):28-45.

29. Prado G, Diaz G Vega y Leon S. Residuos de plaguicidas organoclorados en leche pasteurizada comercializada en Ciudad de México. Arch med vet. 1998;30:55-66.

30. De La Barra M. Determinación del contenido de pesticidas organoclorados en alimentos para ganado bovino, por cromatografía gas-líquido. Valdivia: Universidad Austral de Chile; 1987.

31. Mahieu M. L'evolution de la contamination du lait par les insecticides organochlorés entre 1970 et 1972. Le Lait 1974;54:165-79.

32. Mitchell GE, Wood A.F., Annand C., Sigley E.V. Pesticide residue levels in Queensland pasteurized milks. Aust J Dairy Technol. 1986;42:63-6.

33. Izquierdo P, Allara M, Torres G, García A, Piñero M. Residuos de plaguicidas organoclorados en fórmulas infantiles. Revista Científica. 2004 abril 14 (2):1-13.

34. Gil P. Medicina Preventiva y Salud Pública. Masson Editorial 2004, Barcelona, España.

35. Duarte F, De Castañeda C. Efectos de los plaguicidas en Honduras. Revista Medica Hondureña. 1991;59:155-9. 